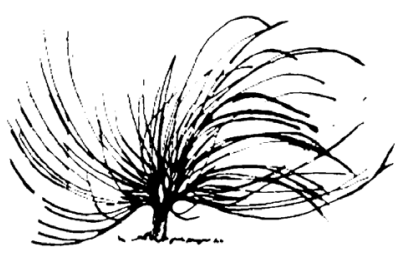

\title{
Una mirada a la epistemología latinoamericana y la mediación didáctica en la enseñanza de las ciencias en general
}

\author{
Luis Diego Mesén Mora \\ Universidad Nacional \\ Costa Rica \\ luis.mesen.mora@una.ac.cr
}

\begin{abstract}
Resumen
El análisis crítico de la epistemología tradicional y latinoamericana logra construir un marco de referencia que ayuda a entender la situación educativa actual. La marcada tendencia globalizadora en los procesos de enseñanza y aprendizaje ha colaborado a aumentar la brecha social desde los tiempos coloniales. El desarrollo cultural autóctono, es la base para crear una conciencia latinoamericana que sustente los procesos educativos en las tradiciones y no en la epistemología occidental impuesta. De esta manera, la pedagogía crítica y emancipadora se convierte en un instrumento para diseñar un proceso de aprendizaje que fomente el desarrollo de habilidades sociales, culturales y científicas que apoyen la evolución país. Con esta base epistemológica, se puede trabajar con una didáctica crítica en la enseñanza de las ciencias, en general, que consienta abrir nuevos modelos de mediación pedagógica que logren una educación integral y equitativa.
\end{abstract}

Recibido: 28 de octubre de 2019. Aprobado: 2 de setiembre de 2020.

http://dx.doi.org/10.15359/rep.16-1.3

1 Químico Industrial, Magíster en Educación con énfasis en Pedagogía Universitaria (UNA). Académico-Investigador Escuela de Química, Universidad Nacional, Costa Rica. 
Palabras clave: didáctica crítica, enseñanza de las ciencias, epistemología latinoamericana, pedagogía crítica, pedagogía tradicional

\begin{abstract}
The critical analysis of traditional and Latin American epistemology allows building a frame of reference that helps to understand the current educational situation. The marked globalization trend in the teaching and learning processes has helped to increase the social gap since colonial times. The development of autochthonous culture permits the creation of a Latin American conscience that allows basing the educational processes on the traditions and not on the imposed Western epistemology. In this way, critical and emancipatory pedagogy allows us to design a learning process that fosters the development of social, cultural, and scientific skills that support the evolution of the country. With this epistemological basis, it is possible to work with a critical didactics in the teaching of science that allows opening new models of pedagogical mediation, achieving a comprehensive and equitable education.
\end{abstract}

Keywords: Latin-American epistemology, critical pedagogy, traditional pedagogy, critical didactics, science education

\title{
Introducción
}

A partir de la independencia de América Latina, se inició un 1 periodo de inestabilidad política, durante el cual se utilizaron $\mathcal{L}$ los procesos educativos como mecanismos de desarrollo para las nuevas naciones, esto se dio como medio de transmisión de valores preestablecidos asociados a la generación de una nueva identidad de estos países, así como de integración nacional para lograr una homogeneidad cultural y social (Hervis, 2017).

La evolución de América Latina ha presentado un marcado componente eurocentrista, en el cual se tienden a dejar de lado las características propias del ser latinoamericano con una fuerte imposición de tradiciones ajenas (Di Filippo, 2007). Esto ha provocado un desarrollo 
desigual, con clases sociales marcadas y una dominancia latente de los grupos poderosos, los cuales cuentan con la mano de obra de los grupos débiles como una de las herramientas de acumulación de riquezas (Pérez y Alemán, 2017).

Este tipo de desarrollo se ve influenciado por la pedagogía tradicional, en la cual se mantiene la importancia de las clases sociales como medio de control (Rivadeneira y Silva, 2017). En este marco de referencia, la educación es el principal mecanismo para mantener las condiciones socio-económicas de los actores sociales, lo cual limita la posibilidad de evolución social para los sectores dominados (Martínez, 2014).

La pedagogía tradicional se basa en diversas teorías, como son la funcionalista, la estructural-funcionalista y la del capital humano (Martínez, 2014). Si bien es cierto, cada una de ellas presenta ciertas diferencias, en todas se fomenta una dominación de las clases menos privilegiadas en manos de las clases altas. Lo anterior por medio de diferentes mecanismos que utilizan a los procesos educativos como herramientas para mantener la estabilidad social, la estratificación de clases y aumentar el dominio social, cultural y económico; de la mano de fundamentos occidentales muy marcados (Cadenas, 2016). Estas teorías están asociadas a un Estado controlador, que busca mantener las condiciones sociales de los más privilegiados, lo que conserva una alta brecha social y pocas posibilidades de desarrollo (Ghiso, 2016).

Este período de control social llevó a la necesidad de liberación y emancipación de las clases sociales oprimidas (Fontana, Liaudat y Baldoni, 2018). De esta manera, se inició el desarrollo de modelos pedagógicos que se basaban en una crítica social fundamentada en la esencia del ser latinoamericano, lo que derivó en el nacimiento de la pedagogía crítica, a la luz de la praxeología y la pedagogía de la liberación, entre otras corrientes actuales que exhortan al cambio social y al desarrollo equitativo (Rivas y Sánchez, 2016).

El análisis del desarrollo epistemológico tradicional y moderno en América Latina, es una de las principales vías para comprender las condiciones de la evolución educativa adecuada, asociadas a una mejora de los procesos de enseñanza y aprendizaje con una fundamentación autóctona, integral, social y equitativa. Este análisis desarrolla una comprensión de las necesidades de las poblaciones actuales para mejorar la mediación pedagógica, con un contexto definido y que resulte en 
un proceso de aprendizaje heurístico, moderno y asociado a las necesidades socioculturales. A partir de esta discusión, se busca la motivación de los académicos especializados en la enseñanza de las ciencias a crear procesos educativos discursivos, menos memorísticos y con un contexto latinoamericano como eje central.

\section{Nuevo panorama de la epistemología latinoamericana}

Gran parte de los modelos educativos actuales se fundamentan en una teoría del conocimiento que no toma en cuenta las raíces ni la idiosincrasia de América Latina. El proceso educativo basado en esta epistemología ajena busca favorecer el mercantilismo y posiciones hegemónicas externas a la cultura latina, donde la escuela se representa como un ente prestador de un servicio (Lander, 2000).

Tal como lo expresa Rodríguez (2017): "el individuo ha sido objetivado en un proceso finito, concluido; resultado de una educación positivista, reduccionista, economicista que se ha entendido como el vehículo instrumental para alcanzar metas económicas compatibles con sus aspiraciones e intereses" (p. 428). Esta visión restringe a la educación como un medio de control social y de crecimiento meramente económico que beneficia a los ya favorecidos y limita las posibilidades de evolución de los más perjudicados socialmente.

Las condiciones desiguales propias del desarrollo latinoamericano están influenciadas por fenómenos como la modernidad, el capitalismo y el eurocentrismo (excluyente de la idiosincrasia autóctona), lo que ratifica la necesidad de una evolución y emancipación que derive en la equidad social (Mendoza, 2010). La modernidad se considera "una salida al atraso de los indios, lo que justificó el uso de la violencia en la conquista y próxima colonización" (Camelo, 2017, p. 113). De esta manera, la imposición cultural derivada de los procesos tanto de conquista como coloniales promovió un desarrollo desigual asociado a los intereses eurocentristas, por lo que la necesidad de métodos educativos orientados hacia el equilibrio social, cultural y económico basado en las raíces culturales americanas es una de las principales herramientas de cambio para lograr las metas futuras (Villalobos, 2015).

La necesidad de mejorar y evolucionar socialmente llevó a los procesos educativos a un acercamiento hacia una academia transmoderna (más allá de la modernidad) que pretende romper los paradigmas impuestos para lograr una equidad social basada en factores autóctonos 
y no impuestos desde el exterior (Fontana, Liaudat y Baldoni, 2018). Dussel (1992, según se cita en Rodríguez, M., 2018) define la transmodernidad como "el nuevo proyecto de liberación de las víctimas de la modernidad" (p. 2), lo que conlleva una mejora de las condiciones socioculturales latinoamericanas.

Este fenómeno sociopolítico, busca una mayor influencia de las corrientes propias, interculturales, tradicionales y emancipadoras lo que deja de lado la imposición europea (Rivas y Sánchez, 2016). De esta manera, se desea alcanzar una liberación de las tendencias epistemológicas del occidente enfocándose en una filosofía del sur (latinoamericana), en donde se procura un desarrollo transmodernista basado completamente en la epistemología autóctona (Mendoza, 2010).

Esta filosofía del sur genera una educación liberadora, que se enfoca en lo que se puede y se necesita crear, con base en la discusión educativa integral (Fontana, Liaudat y Baldoni, 2018). Esta tendencia liberadora, debe alejarse lo más posible de la epistemología eurocéntrica, ya que esta intenta reproducir el sistema actual, de manera que la escuela se encargue de enseñar lo que debe ser transmitido, con énfasis en mantener las condiciones desiguales (Cerletti, 2008). Este proceso es llamado por Fontana, Liaudat y Baldoni (2018), como la "desoccidentalización del conocimiento" (p. 3), y tiene como principal objetivo convertirse en una alternativa eficaz para superar la "epistemología occidentalcéntrica imperial" (p. 4).

Dentro de los principales objetivos de la epistemología latinoamericana, está poner en tela de dudas la concepción filosófica y científica heredada o impuesta por los criterios occidentales, lo que conlleva a validar el conocimiento asociado a los pueblos latinoamericanos que procura la academia transmoderna como meta principal (Retamozo, 2011).

Estas tendencias latinoamericanas, cuentan como uno de los principales exponentes a Enrique Dussel, quien en su teoría de la filosofía de la liberación identifica el potencial para emanciparse de aquellos actores que se encuentran en un estado de exclusión social (Bauer, 2016). Se centra en motivar a los sectores sociales desfavorecidos a crear una resistencia hacia el sistema vigente para generar una praxis que pueda transformar el marco sociocultural (Ghiso, 2016), de esta manera: 
La Filosofía de la Liberación se ha constituido como un proyecto intelectual de Latinoamérica, no solamente producido en la región, puesto que ha promovido un pensar autónomo de los centros de poder mundial, reconociendo el pensamiento latinoamericano desde la opresión y la dependencia. (Cabaluz, 2016, p. 71)

De esta manera, el modelo político de Dussel se considera lo más cercano a la perfección que se pueda alcanzar en este momento histórico, donde los actores excluidos, deben irrumpir por medio de la crítica, la discusión y el análisis en espacios de cambio del sistema tradicional (Mendoza, 2010). Este punto de máxima perfección es denominado transmodernidad, la cual "contrario a lo que señalan erróneamente, no trata de volver sobre un pasado perfecto, de lo que trata es de volver a pensar todo, sin desconocer la tradición (Rodríguez, A., 2018, p. 84). Es una meta social, un objetivo de vida al cual queremos llegar y por el cual nos desarrollamos, en donde, el camino que debemos seguir para lograr acercarnos al máximo punto de la integralidad del individuo social es la pedagogía (Mendoza-Álvarez, 2016).

En palabras de Grosfoel (2017, según se cita en Rodríguez, A., 2018):

Tampoco se trata de romantizar el pasado y volver a un pasado idílico premoderno, lo cual es imposible. Lo que se propone es un proyecto político más allá de la Modernidad o como dice el filósofo de la liberación latinoamericana Enrique Dussel, un proyecto hacia la "Transmodernidad" desde la diversidad epistémica del mundo. (p. 84)

Esta nueva tendencia pedagógica busca que las víctimas del sistema tradicional asuman la crítica hacia este y otras instituciones que mantienen los privilegios a las clases dominantes al promover la equidad social por medio de la educación (Fernández, 2013). De la mano de las teorías de Dussel, se desarrollaron diversas corrientes de pensamiento educativo como lo es la pedagogía de la liberación de Paulo Freire:

Insisto en que la educación en general es una educación de respuestas, en lugar de ser una educación de preguntas, que es la 
única educación creativa y apta para estimular la capacidad humana de asombrarse, de responder al asombro y resolver los verdaderos problemas esenciales, existenciales, y el propio conocimiento. (Villalobos, 2015, p. 98)

Freire denuncia cómo los actores que pertenecen a las clases sociales elitistas, desean mantener su estatus y control sociopolítico, deja claro cómo estas clases privilegiadas desean mantener este control por medio de una falsa generosidad a las clases oprimidas, siendo estas las responsables de conservar el orden social injusto (Martínez, 2014). "La educación verdadera es praxis, reflexión y acción del hombre sobre el mundo para transformarlo" (Freire, 2009, p. 7).

La educación debe ser parte de un proceso emancipador, el cual debe promover un mundo más justo y motivar al desarrollo de un proceso solidario que derive en una mayor equidad con los grupos desfavorecidos (Carreño, 2009). Como educadores, es importante conocer el trasfondo teórico para que las decisiones en el aula sean adecuadas (Dorrego, 2016). Debemos tomar en cuenta las raíces culturales que hemos dejado de lado y no apegarnos a sistemas impuestos y tradicionalistas (Escorza y Aradillas, 2013). Fomentar el desarrollo educativo basado en nuestra idiosincrasia, en las necesidades socioculturales, en la dialéctica como herramienta de desarrollo que busca que la educación trascienda más allá de un ejercicio memorístico, son parte de los principales retos que se deben asumir como una sociedad latinoamericana unida con miras a la evolución esperada (Fernández, 2013).

\section{Pedagogía tradicional y educación}

Basado en el liberalismo europeo a principios del siglo XIX, el Estado no tenía dentro de sus prioridades la intervención en las nuevas naciones, pero debido al requerimiento de una identidad nacional, se promovieron iniciativas educativas con el fin de construir esta noción país (Hervis, 2017). Estos primeros esfuerzos del Estado mantuvieron las políticas occidentales impuestas, lo que derivó en un sistema educativo rígido, en el cual se busca que las clases altas mantengan de manera activa su estrato social (Estermann, 2014).

Este sistema tradicional presenta características muy marcadas, como lo es la asistencia obligatoria al cumplir con un horario, así como una serie de directrices específicas, además del análisis de 
contenidos que promueven estudiantes sin identidad propia, una evaluación estandarizada y un proceso educativo carente de contexto social (Méndez-Giménez, Fernández-Río y Méndez-Alonso, 2015). Así pues, Mendoza (2010) denomina estas características propias del sistema educativo tradicionalista como el "hito de santidad" (p. 11).

La epistemología de la pedagogía tradicional está basada en diversas teorías, una de las principales es la funcionalista, la cual establece la necesidad de las clases sociales, se enfatiza que deben existir personas con diferentes funciones definidas, algunas privilegiadas y otras desfavorecidas (Cadenas, 2016). Pero deja claro que esta diferencia de clases es vital para el buen funcionamiento de la sociedad, en donde, la educación tiene un papel de control y adaptación de los individuos a su realidad social (Elster, 2015). Los procesos educativos deben desarrollar las habilidades requeridas por su entorno para que los individuos cumplan su función, "la educación es la acción ejercida por las generaciones adultas sobre aquellas que no han alcanzado todavía el grado de madurez necesario para la vida social" (Durkheim, 2009, p. 49), de esta manera se mantiene el control y estado social aceptado.

La pedagogía tradicional está relacionada con otras dos teorías, la estructural-funcionalista y la del capital humano, todas mantienen la idea de un Estado controlador y un sistema educativo como herramienta para lograr este fin (Mendoza, 2010). La teoría estructural-funcionalista hace referencia a la necesidad de los estratos, al hacer una analogía entre los seres vivos y el Estado (Reyes, Téllez, y Orbeas, 2018). Mientras que la teoría de capital humano fomenta el desarrollo comercial de la educación, lo que da pie a la privatización de esta y a la preparación de los alumnos para cumplir sus funciones específicas con un beneficio económico adjunto, además limita al máximo la investigación, ya que esta no genera un beneficio económico sino conocimiento (Pérez-Fuentes, y Castillo-Loaiza, 2016).

Estas teorías promueven un sistema educativo autoritario, donde el profesor tiene el control y es el dueño del conocimiento, el cual es transmitido al estudiante para que este acepte y cumpla su función social establecida (Méndez et al., 2015). De esta manera, se puede hacer una relación directa entre la pedagogía tradicional y la teoría conductista del aprendizaje.

Esta teoría se basa en el control de la conducta como una de sus características más sobresalientes, además de minimizar los 
sentimientos y maximizar su estudio experimental (Peña, 2010). De esta forma, se ignora por completo la crítica y la capacidad analítica de los estudiantes, mientras se hace énfasis en que las consecuencias son las encargadas de mantener los comportamientos esperados de los individuos, así es como, las situaciones educativas se basan en el estímulo y respuesta, con una férrea defensa de la sistematización de los conocimientos transmitidos (Pellón, 2013). Como pilar fundamental, el conductismo, requiere que el alumno se capaz de cumplir los deberes que le fueron asignados y que fueron aprendidos durante el proceso educativo, lo que confirma que esta teoría es fundamental para el desarrollo de la pedagogía tradicional (Cabezas, 2011).

Para conductistas modernos, esta teoría tiene un futuro prometedor siempre que no se utilice como mecanismo de condicionamiento operante como herramienta de modificación y adquisición de conocimiento, sino que se deben explorar nuevos conceptos y caminos para lograr cabida en el contexto actual (Peña, 2010). Lo que evidencia la necesidad de evolucionar para lograr un desarrollo social contextualizado y actual.

\section{Emancipación de las clases desfavorecidas: la pedagogía crítica}

La pedagogía crítica nace en contraposición a las teorías centradas en el occidentalismo europeo, en donde la educación es considerada como un sistema bancario y el alumno es un simple acumulador de los conceptos aprendidos, mientras el profesor es el dueño del conocimiento (Estermann, 2014). Dentro de las principales consecuencias de la pedagogía tradicional, recae el aumento de la brecha social por medio de los modelos educativos heredados, la cual busca mantener el statu quo de las clases privilegiadas (Reyes et al., 2018).

El deseo de mejorar las condiciones de las clases oprimidas llevó a diversos esfuerzos por crear una escuela basada en la idiosincrasia latinoamericana, con la cual las clases sociales desfavorecidas puedan luchar por la autosuperación en un contexto sociocultural enmarcado en sus propias necesidades (Carreño, 2009). A raíz de esta situación de dominancia, nace la pedagogía crítica, la cual no se basa en la transmisión del conocimiento, sino en la construcción de este mediante un punto de vista problematizador y contextualizado (Alvarado, 2007). Según Giroux (2016), la pedagogía crítica tiene como uno de sus principales fines: 
Desafiar al neoliberalismo y a otras tradiciones antidemocráticas incluyendo la creciente criminalización de los problemas sociales tales como la falta de hogar, al mismo tiempo que restablecen un proyecto democrático radical que provee las bases para imaginar una vida más allá del mundo de los sueños del capitalismo. (p. 14)

Este esfuerzo implica una independencia de la epistemología occidental y eurocentrista que ha dominado las clases educativas por muchos años (Santos, 2017). A raíz de esta necesidad, los sistemas educativos se enfocan en no solo explicar los hechos, sino en crear conciencia en lo que debe ser (Rodríguez, Álvarez y Bernal, 2016). Para que la liberación de las clases oprimidas sea efectiva, estas deben entender su responsabilidad, no únicamente aceptar las condiciones en las que se desenvuelven sino luchar por mejorar su situación, es en este punto donde la pedagogía crítica actúa e inicia el cambio en el paradigma antropocéntrico occidental (Alvarado, 2007).

Este enfoque a la pedagogía crítica no reduce la práctica educativa al dominio de las metodologías, sino que enfatiza, en cambio, la importancia de comprender lo que realmente ocurre en las aulas y en otros contextos educativos a través de preguntas tales como ¿cuál es la relación existente entre el aprendizaje y el cambio social?, ¿qué tipo de conocimiento tiene más valor?, ¿qué significa saber algo?, y ¿en qué dirección debería uno desear? (Giroux, 2016, p. 14)

Tal como lo menciona Mendoza (2010), la pedagogía crítica busca romper el hito de santidad, asociado al occidentalismo ideológico que ha dominado el desarrollo latinoamericano. Esta ruptura implica una problemática social que se debe resolver desde los inicios del sistema educativo. Por ejemplo, se necesita una pedagogía liberadora en las aulas, pero por lo general en ellas se encuentran docentes que, en algunos casos, forman parte de las clases oprimidas (Santos, 2017). La importancia de un sistema educativo basado en el contexto social, los sentimientos, la experiencia de los actores sociales y el deseo de superación son vitales en todos los estratos educativos, para fomentar los procesos emancipadores de forma integral (Rodríguez et al., 2016). 
Diversas teorías de aprendizaje son consecuentes con el modelo pedagógico discursivo. En el cognitivismo, si es importante el sentir del estudiante y con el profesor como facilitador, en este marco educativo, la realimentación tiene una función de guía y consolida el conocimiento con su relación social (Castañón, 2017). En el caso del constructivismo, la elaboración del conocimiento mediante nuevas experiencias es de vital importancia; con ayuda de un profesor mediador, se fomenta un proceso de educación continua, no impositiva (Fernández, 2016).

Todos los seres humanos tienen la capacidad de aprender y asociar este aprendizaje no solo como mecanismo de generación de riquezas, sino como una herramienta de evolución, liberación, discusión, reflexión y análisis (Fontana, Liaudat y Baldoni, 2018). Estos elementos son parte de las ideas fundamentales para que el proceso educativo sea praxeológico, contextualizado y sea una guía hacia la academia posmoderna en una sociedad culturalmente equitativa (Freire, 2009).

\section{Didáctica crítica: nueva visión para la enseñanza de las ciencias}

La didáctica como mecanismo de mediación educativa debe ser concordante con el modelo pedagógico aplicado en las clases (Martínez et al., 2012). Si se utiliza un modelo heurístico con un fuerte componente analítico, debemos procurar la aplicación de una didáctica crítica, que logre que los alumnos construyan, compartan, socialicen y sistematicen de manera que el conocimiento sea significativo y estrechamente ligado con la pedagogía emancipadora (Osborne, 2009). De esta manera y según lo expresado por Carrasco, Baldivieso y Di Lorenzo (2016), el desarrollo investigativo autóctono es vital para el desarrollo cultural:

América Latina requiere ampliar su mirada en la formación de investigadores, situados en la sociedad latinoamericana actual, que tiene su propia forma de evolucionar, diferente a la europea o asiática por decir, pues es fundamental que estos investigadores no sólo puedan observar lo que ocurre en el momento. (p. 16)

La enseñanza de las ciencias siempre ha estado marcada por un modelo positivista y memorístico, que no toma en cuenta el contexto de los estudiantes y, por lo general, se centra en la magistralidad de las clases, transmisión del mensaje educativo y resolución mecánica de preguntas (Valle y Álvarez, 2016). Pero a la luz de las tendencias 
liberadoras latinoamericanas, el estudio de las ciencias debe enfocarse no solo en la memoria y transmisión, sino la crítica y desarrollo de habilidades cognitivas que generen profesionales socialmente comprometidos (Martínez et al., 2012). Esta es una de las herramientas con las cuales podemos evolucionar en la praxis docente y en el progreso sociocultural.

Las teorías contemporáneas desarrollan las destrezas con el fin de no utilizar la educación como un filtro de gente según sus habilidades y talentos, sino como un ente estimulador y desarrollador de estas, de la mano de pensar y aprender (Osborne, 2009). Los alumnos pueden aprender mejor por medio de actividades didácticas bien construidas, como son las investigaciones y discusión de estas mediante la mediación docente (Martínez et al., 2012).

El desarrollo de habilidades como la resolución de problemas científicos, se puede abordar mejor dentro de un marco crítico, donde la necesidad de un esfuerzo cognoscitivo va de la mano con los rasgos y el contexto social de la persona, lo que facilita el resultado y la construcción del conocimiento (Cruz y Elers, 2019). Para que esta filosofía discursiva tenga el éxito esperado, es muy importante fomentar la evolución y capacitación docente (Cuervo et al., 2011). La necesidad de una docencia eficiente, según Reyes y Garritz (2006), va de la mano de la "validez ecológica" (p. 1180), la cual se enfoca en un aprendizaje basado en las condiciones de la clase y cómo se afectará con el contexto integral de los estudiantes.

Esta validez ecológica es vital para tomar decisiones importantes en el proceso de enseñanza y aprendizaje, al desarrollar un contexto integrado con las necesidades reales y el perfil profesional de los estudiantes graduados (Fernández, 2016). Actualmente, se pueden analizar diversos ejemplos de cómo se integra una metodología más discursiva y participativa en los procesos de aprendizaje científico. A continuación, se presentarán dos casos, el primero de la Universidad Nacional de Costa Rica y el segundo de la Universidad de Costa Rica, lo que reafirma la importancia de una educación integradora y contextualizada en el nivel nacional.

En primera instancia, se presenta el caso de la carrera Ingeniería en Gestión Ambiental, impartida en la Universidad Nacional, la cual ofrece el curso Laboratorio de Química Orgánica Ambiental. Durante muchos años, este curso fue impartido de manera tradicional, mediante prácticas de laboratorio guiadas y preparadas que comprueban los 
principios de la química orgánica, pero sin relación con las necesidades propias de sus estudiantes. A partir del año 2018, se realizó un cambio metodológico, en donde ya no se resolvieron prácticas diseñadas para tener resultados positivos inmediatos que comprueben los principios teóricos; sino que se realizaron muestreos de zonas con alto riesgo de contaminación ambiental, para ser analizadas y preparar un informe técnico que diagnostique las características de estas. Lo que conlleva una validez ecológica directa con las condiciones actuales de análisis (Reyes y Garritz, 2006). Es claro que estas muestras presentan una mayor dificultad de tratamiento debido a su origen, pero además contextualizan la situación social, económica y cultural de las comunidades visitadas, lo cual logra un aprendizaje integral entre el desarrollo técnico-científico y el aporte social a la calidad de vida (Cuervo et al., 2011).

Así es como, el quehacer de los profesionales en Gestión Ambiental, de la mano del conocimiento creado por medio de la investigación y la contextualización, se convierte en un importante factor para desarrollar un proceso educativo novedoso integral, con una aplicación directa que genere una mejoría en las condiciones del entorno comunal basado en aprendizaje investigativo (Rodríguez et al., 2016).

El segundo caso de estudio confirma la importancia del aprendizaje contextualizado y arraigado en las necesidades autóctonas; así como que el desarrollo industrial debe estar ligado a la concientización ecológica y social. Lo anterior es un ejemplo que se puede analizar en el transcurso del curso Laboratorio de procesos industriales 2 de la carrera de Química de la Universidad de Costa Rica. Este curso se basa en generar habilidades de producción industrial con una aplicación ligada a los requerimientos sociales de alguna comunidad o de una institución.

Es así como, por medio de la investigación aplicada en la tecnología química, los estudiantes del curso diseñaron una serie de productos que fueron donados al hogar Montiel de niñas en riesgo social; esta integración de los procesos químicos con las necesidades sociales fortalece el conocimiento con el contexto cultural y orienta el aprendizaje de manera integral (Vargas, 2019a). Otro de los propósitos del curso recae en la sostenibilidad ambiental, en este caso la creación de un detergente basado en desechos de cascara de huevo, y así suplir las necesidades de limpieza de los conserjes de la universidad, que mantiene una intrínseca relación con la conservación ecológica por medio de productos biodegradables (Vargas, 2019b). 
En ambos casos, el uso de técnicas de mediación pedagógica discursivas, investigativas y entrelazadas con el contexto social y la didáctica crítica, genera que los estudiantes logren un aprendizaje significativo con una relación muy cercana a la idiosincrasia de sus comunidades. Así como una relación proporcional con la validez ecológica, basados en acciones concretas que beneficien el entorno educativo (Zambrano, 2018).

Como seres humanos, la relación social es parte vital del quehacer diario, esta debe ser intencional, recíproca, acumulativa, de apoyo y colectiva (Valle y Álvarez, 2016). Este aprendizaje cooperativo no memorístico se basa en procesos epistémicos como discutir, criticar, argumentar, explicar, entre otros; de esta manera se establece un desarrollo más activo (Osborne, 2009). Este tipo de procesos son menos utilizados en la enseñanza de las ciencias, lo que presenta uno de los principales retos por afrontar, pero de los más significativos para realzar la importancia de la discusión en el conocimiento científico (Rivera, 2017). Y aunque su implementación no es fácil ni asociada a la tradicionalidad educativa de los modelos actuales, es completamente viable fomentar una educación crítica, integral y analítica para así poder colaborar con el desarrollo de profesionales completos.

\section{Conclusiones}

El desarrollo de los pueblos latinoamericanos siempre ha estado influenciado por una epistemología occidental, en la cual las necesidades de los pueblos basadas en su tradición, han sido ignoradas a través del tiempo, lo que fomenta una separación de clases muy marcada, lo que lleva a un desequilibrio social cada vez más grande.

Este sistema de favorecimiento para ciertas clases específicas se fundamenta en la pedagogía tradicional, la cual, por medio de herramientas conductuales, busca una aceptación del papel sociocultural y una repetición de individuos, con la finalidad de perpetuar las condiciones de las clases sociales altas debido al trabajo de los menos favorecidos. De esta manera, la escuela se convierte en un mecanismo de control y no un medio de evolución.

Estas situaciones llevaron a promover una pedagogía de la liberación, la cual se fundamenta en la crítica de la crítica, la discusión, las tradiciones autóctonas y el deseo de superación de las clases oprimidas por medio de los procesos educativos. En este sistema, la educación no es un medio de control de clases, sino una herramienta emancipadora 
asociado al desarrollo social, económico y cultura de las clases bajas y, por ende, la equidad social esperada en las teorías posmodernas.

La enseñanza de las ciencias se ha enmarcado en un sistema conductista-positivista que logra la formación de profesionales iguales, pero con pocas habilidades de construcción y discusión. Basados en las teorías emancipatorias, es de vital importancia fomentar el uso de una didáctica crítica, para no solo desarrollar profesionales expertos en las áreas científicas, sino que tengan la capacidad de integrarse socialmente y contribuir de manera directa en la evolución cultural de su entorno.

\section{Referencias}

Alvarado, M. (2007). José Martí y Paulo Freire: aproximaciones para una lectura de la pedagogía crítica. Revista Electrónica de Investigación Educativa, 9(1), 1-19. Recuperado de https://www. redalyc.org/pdf/155/15590104.pdf

Bauer, C. (2016). Filosofía social crítica de los medios hegemónicos de comunicación Sistema mundo colonial-neocolonial, aldea global y función social del radiorreceptor en el proceso de descolonización y liberación de Argelia. Revista Brasileira de História da Midia, 5(2). 134-152. Recuperado de https://revistas.ufpi.br/index.php/rbhm/article/view/4728/3089

Cabaluz, J. (2016). Pedagogías críticas latinoamericanas y filosofía de la liberación: potencialidades de un diálogo teórico-político. Educación y Educadores, 19(1), 67-88. Recuperado de https:// dialnet.unirioja.es/servlet/articulo?codigo $=5558825$

Cabezas, J. (2011). Consideraciones preliminares entorno del pensamiento pedagógico del Profesor. Cuadernos de Lingüística Hispánica, 18, 95-112. Recuperado de https://revistas.uptc.edu.co/ revistas/index.php/linguistica_hispanica/article/view/435

Cadenas, H. (2016). La función del funcionalismo: una exploración conceptual. Sociologias, 18(41), 196-2014. Recuperado de https://www.seer.ufrgs.br/sociologias/article/view/62244

Camelo, D. (2017). Enrique Dussel y el mito de la modernidad. Cuadernos de Filosofia Latinoamericana, 38(116), 97-115. Recuperado de https://dialnet.unirioja.es/servlet/articulo? codigo $=6268333$

Carrasco, S., Baldivieso, S., y Di Lorenzo, L. (2016). Formación en investigación educativa en la sociedad digital. Una experiencia innovadora de enseñanza en el nivel superior en el contexto 
latinoamericano. Revista de Educación a Distancia, 48, 1-19. Recuperado de https://revistas.um.es/red/article/view/253481

Carreño, M. (2009). Teoría y práctica de una educación liberadora: el pensamiento pedagógico de Paulo Freire. Cuestiones Pedagógicas, 20, 195-214. Recuperado de https://institucional.us.es/revistas/cuestiones/20/art_10.pdf

Castañon, G. (2017). O cognitivismo é um humanismo. Psicologia Argumento, 25(48), 51-64

Cerletti, A. (2008). La enseñanza de la filosofia como problema filosófico/The teaching of philosophy as a philosophical problema (Vol. 2). Buenos Aires: Libros del Zorzal.

Cruz, M., y Elers, M. (2019). Estrategia de los estudiantes para resolver problemas en Química Física: una experiencia pedagógica. Pedagogía Universitaria, 24(1), 43-54.

Cuervo, Á., Armenta, J., Martínez, M., López, R., y Lozoya, S. (2011). Necesidades de capacitación de docentes de educación básica en el uso de las TIC. Pixel-Bit. Revista de Medios y Educación, 39, 211-223. Recuperado de https://idus.us.es/handle/11441/45678;jsessionid=396732C64380EB44D61A9BC2F3D85DA3?

Di Filippo, A. (2007). La Escuela Latinoamericana del Desarrollo: Tensiones epistemológicas de un movimiento fundacional. Cinta de Moebio. Revista de Epistemología de Ciencias Sociales, 29, 56-68. Recuperado de http://www.facso.uchile.cl/publicaciones/ moebio/29/difilippo.pdf

Dorrego, E. (2016). Educación a distancia y evaluación del aprendizaje. RED: Revista de Educación a Distancia, 50, 10-20. Recuperado de https://www.um.es/ead/red/M6/dorrego.pdf

Durkheim, E. (2009). Educación y sociología. México: Ediciones Yucatán.

Elster, J. (2015). Marxismo, funcionalismo y teoría de juegos: Alegato en favor del individualismo metodológico. Sociológica México, 2, 3-28. Recuperado de http://www.sociologicamexico.azc.uam. $\mathrm{mx} /$ index.php/Sociologica/article/view/1094

Escorza, Y. y Aradillas, A. (2013). Teorías del aprendizaje en el contexto educativo. Monterrey: Editorial Digital de Tecnológico de Monterrey.

Estermann, J. (2014). Colonialidad, descolonización e interculturalidad. Apuntes desde la Filosofía Intercultural. Polis. 
Revista Latinoamericana, 38, 1-19. http://dx.doi.org/10.4067/ S0718-65682014000200016

Fernández, E. (2016). Aprendizaje constructivista para el análisis de estructuras mediante el uso de un entorno virtual/Constructivist learning for the analysis of structures by using a virtual environment. Revista Tecnocientifica URU, 9, 41-50.

Fernández, I. (2013). Redefinición de los alcances de la pedagogía de la liberación en sus dimensiones ética, política y cultural. Historia de la Educación. Anuario, 13(2), 1-6. Recuperado de http://ppct. caicyt.gov.ar/index.php/anuario/article/view/2354

Fontana, J., Liaudat, S., y Baldoni, C. (Noviembre, 2018). La descolonización epistemológico-pedagógica será desobediente o no será. Trabajo presentado en, VIII Conferencia Latinoamericana y Caribeña de Ciencias Sociales y I Foro Mundial del Pensamiento Crítico (CLACSO). Buenos Aires, Argentina.

Freire, P. (2009). La educación como práctica de la libertad. México: Siglo XXI Editores.

Ghiso, A. M. (2016). Profesionalización de pedagogos sociales en Latinoamérica. Otra lectura a los tránsitos entre la educación popular y la pedagogía social. Agora USB, 16(1), 63-75. Recuperado de http://www.scielo.org.co/scielo. php?script $=$ sci_abstract\&pid $=$ S1657-80312016000100003

Giroux, H. (2016). La pedagogía crítica en tiempos oscuros de Henry Giroux. Obert, G. y Eliggi, G. (Traductoras). Praxis Educativa, 17(2), 13-26. Recuperado de https://cerac.unlpam.edu.ar/index. $\mathrm{php} / \mathrm{praxis} /$ article/view/1648

Hervis, E. (2017). La educación en América Latina: Desarrollo y perspectivas. Revista Electrónica Actualidades Investigativas en Educación, 17(2), 1-23. DOI: 10.15517/aie.v17i1.28147

Lander, E. (2000). Ciencias sociales: saberes coloniales y eurocéntricos. La colonialidad del saber: eurocentrismo y ciencias sociales. Perspectivas latinoamericanas. Buenos Aires: CLACSO.

Martínez, R. (2014). Pedagogía tradicional y pedagogía crítica. Pensamiento crítico n.1.: México: Doble Hélice Ediciones-Instituto Latinoamericano de Pedagogía Crítica, col.

Martínez, T., Joaquín, D., Josep L., Menargues, A., y Romo G. (2012). La integración de los trabajos prácticos en la enseñanza de la química como investigación dirigida. Educación Química, 23 (Supl. 
1), 112-126. Recuperado de http://www.scielo.org.mx/scielo. php?script=sci_abstract\&pid=S0187-893X2012000500004\&ln$\mathrm{g}=\mathrm{es} \& \mathrm{nrm}=\mathrm{iso}$

Méndez-Giménez, A., Fernández-Río, J., y Méndez-Alonso, D. (2015). Modelo de educación deportiva versus modelo tradicional: efectos en la motivación y deportividad. Revista Internacional de Medicina y Ciencias de la Actividad Física y del Deporte/International Journal of Medicine and Science of Physical Activity and Sport, 15(59), 449-466. Recuperado de http://cdeporte.rediris.es/revista/revista59/artmodelo612.htm

Mendoza-Álvarez, C. (2016). La teología de la liberación en contexto posmoderno en América Latina y el Caribe. Perspectiva Teológica, 48(2), 97-115. Recuperado de https://www.researchgate. net/publication/307558664_LA_TEOLOGIA_DE_LA_LIBERACION_EN_CONTEXTO_POSMODERNO_EN_AMERICA_LATINA_Y_EL_CARIBE

Mendoza, B. (2010). La epistemología del sur, la colonialidad del género y el feminismo latinoamericano. Aproximaciones críticas a las prácticas teórico-políticas del feminismo latinoamericano. Buenos Aires, Argentina. Consejo Latinoamericano de Ciencias Sociales.

Osborne, J. (2009). Hacia una pedagogía más social en la educación científica: el papel de la argumentación. Educación Química. 20(2), 156-165. Recuperado de https://www.sciencedirect.com/ science/article/pii/S0187893X18300223

Pellón, R. (2013). Watson, Skinner y Algunas Disputas dentro del Conductismo. Revista Colombiana de Psicología, 22(2), 389399. Recuperado de http://www.scielo.org.co/pdf/rcps/v22n2/ v22n2a12.pdf

Peña, T. (2010). ¿Es viable el conductismo en el siglo XXI? Liberabit, 16(2), 125-130. Recuperado de https://www.researchgate.net/profile/Telmo_Correal/publication/262756458_Es_viable_el_conductismo_en_el_siglo_XXI/links/0912f508f4b540ef59000000/ Es-viable-el-conductismo-en-el-siglo-XXI.pdf

Pérez, M., y Alemán, A. (2017). La polarización latinoamericana en la contemporaneidad/Political Polarization in Today's Latin America. Revista Economía y Desarrollo, 158(2), 63-75. 
Pérez-Fuentes D., y Castillo-Loaiza J. (2016). Capital humano, teorías y métodos: importancia de la variable salud. Economía, Sociedad y Territorio, 16(52), 651673. Recuperado de http://www.scielo.org.mx/scielo. php?script $=$ sci_arttext\&pid $=$ S1405-84212016000300651

Retamozo, M. (2011). Sujetos políticos: teoría y epistemología. Un diálogo entre la teoría del discurso, el (re) constructivismo y la filosofía de la liberación en perspectiva latinoamericana. Ciencia Ergo Sum, 18(1), 81-89. Recuperado de https://www.redalyc.org/ articulo.oa? id $=10416528011$

Reyes, F. y Garritz, A. (2006). Conocimiento pedagógico del concepto de "reacción química" en profesores universitarios mexicanos. Revista Mexicana de Investigación Educativa, 11(31), 11751205. Recuperado de http://www.scielo.org.mx/scielo.php?script=sci_arttext\&pid=S1405-66662006000401175\&lng=es\&n$\mathrm{rm}=\mathrm{iso}$

Reyes, Y., Téllez, M., y Orbeas, G. (2018). Modalidad educativa no formal como expresión de la educación para el desarrollo local. EduSol, 18(63), 93-106. Recuperado de https://dialnet.unirioja. es/servlet/articulo?codigo $=6572871$

Rivadeneira, E., y Silva, R. (2017). Aprendizaje basado en la investigación en el trabajo autónomo y en equipo. Negotium, 13(38), 5-16. Recuperado de http://www.revistanegotium.org.ve/pdf/38/ art1.pdf

Rivas, A., y Sánchez, B. (2016). Políticas y resultados educativos en América Latina: un mapa comparado de siete países (20002015). RELIEVE. Revista Electrónica de Investigación y Evaluación Educativa, 22(1), 1-30. http://dx.doi.org/10.7203/ relieve.22.1.8245

Rivera, D. (2017). Propuesta didáctica para la enseñanza del concepto célula a partir de su historia y epistemología. Tecné Episteme y Didaxis TED, 900-907. Recuperado de http://revistas.pedagogica.edu.co/index.php/TED/article/view/4831

Rodríguez, A. (2018). Una aproximación al proyecto de Transmodernidad de Enrique Dussel. INTERdisciplina, 6(16), 83-103. http:// dx.doi.org/10.22201/ceiich.24485705e.2018.16.65636

Rodríguez, J., Álvarez, E. y Bernal, A. (2016). Caracterización de una pedagogía de la teología en perspectiva 
crítico-liberadora. Reflexiones desde la pedagogía crítica y la teología de la liberación. Albertus Magnus, 7(2), 213-237. DOI: 10.15332/s2011-9771.2016.0002.02

Rodríguez, M. (2017). Currículum, educación y cultura en la formación docente del siglo XXI desde la complejidad. Educación y Humanismo, 19(33), 425-440. Recuperado de https://dialnet.unirioja. es/servlet/articulo?codigo $=6395389$

Rodríguez, M. (2018). Interrelación matricial epistémica inherente a los elementos del patrimonio cultural, la identidad y ciudadanía en la sociedad transmoderna. Visión Educativa, 12(25), 1-86. Recuperado de http://iunaes.mx/inicio/wp-content/uploads/2018/04/ Revista-Visi\%C3\%B3n-IUNAES-No.-25.pdf\#page=7

Santos, J. (2017). Aportes para una pedagogía crítica nuestroamericana: identificando el núcleo conceptual del pensamiento político-pedagógico de Paulo Freire. Revista Pedagógica, 19(41), 80-95. Recuperado de https://bell.unochapeco.edu.br/revistas/index. php/pedagogica/article/view/3801

Valle, S., y Álvarez, J. (2016). Didáctica para la Educación Superior. Alternativas ante la problemática docente en la educación de las ciencias duras. Perspectivas Docentes, 58, 5-12. Recuperado de http://ri.ujat.mx/handle/20.500.12107/2643

Vargas, M. (10 de junio de 2019a). Estudiantes de Química de la UCR crean jabón y desinfectante que ayudarán a hogar de niñas en riesgo social. La Nación. Recuperado de https://www.nacion.com/ciencia/aplicaciones-cientificas/estudiantes-de-quimica-de-la-ucr-crean-jabon-y/ Q5C6EJIUMFGFFACQCKELZHPL64/story/

Vargas, M. (14 de octubre de 2019b). Estudiantes de la UCR crean detergente con cascaras de huevo. La Nación. Recuperado de https://www.nacion.com/ciencia/aplicaciones-cientificas/estudiantes-de-la-ucr-crean-detergente-con/ JFQ4NQ6CSJDDLPHBMAPX6UFXQQ/story/

Villalobos, D. (2015). Planteamientos Pedagógicos Relacionados Con Las Ciudadanías Propias En La Pedagogía Crítica De Paulo Freire. El Ágora USB, 15(1), 195-215. https://doi. org/10.21500/16578031.10

Zambrano, A. (2018). Naturaleza y diferenciación del saber pedagógico y didáctico. Pedagogía Y Saberes, 50, 75-84. https://doi. org/10.17227/pys.num50-9500 